\title{
Propriedades biomecânicas do poli-éter- éter-cetona (PEEK) e sua aplicação na clínica odontológica: uma revisão de líteratura
}

\section{Biomechanical properties of polyether-ether- ketone (PEEK) and its application in dental clinic: a literature review}

\author{
Igor Adolfo Gonçalves Galvão' (1) \\ Phelipe Cruz de Carvalho² \\ Raquel Sousa Feitosa ${ }^{3}$ (D) \\ Ermeson Inácio de Sousa ${ }^{4}$ (D) \\ Manasses Tercio Vieira Grangeiro 5 (D) \\ Viviane Figueiredo ${ }^{6}$ (D)
}

\begin{abstract}
${ }^{1-4}$ Centro Universitário Leão Sampaio (Juazeiro do Norte). Ceará, Brasil. igorggalvao@gmail.com, pheliper7@gmail.com, raquelfeitosaphc@gmail.com, ermeson_deus@hotmail.com ${ }^{5}$ Universidade Estadual Paulista (São José dos Campos). São Paulo, Brasil. terciomanasses@gmail.com ${ }^{6}$ Autora para correspondência. Universidade Federal do Pernambuco (Recife). Pernambuco, Brasil. vivi_mfigueiredo@yahoo.com.br
\end{abstract}

RESUMO | OBJETIVO: Revisar a literatura sobre o biomaterial poli-éter-éter-cetona (PEEK) enfatizando as propriedades mecânicas e aplicações na odontologia. MATERIAL E MÉTODO: Foi realizada uma busca nas bases de dados eletrônicas PubMed, Scielo e Bireme, utilizando os termos de busca: Materiais Dentários, Implantes Dentários, Prótese Dentária. Os critérios de inclusão e exclusão foram estudos in sílico, in vitro, revisão sistemática da literatura que aborda as variáveis em estudo, idioma em inglês e português. RESULTADOS: PEEK vem ganhando destaque importante na odontologia, uma vez que possui uma relação de alta resistência a peso, propriedades elásticas similares ao osso humano, capacidade de absorver choque durante a mastigação, alta resistência ao desgaste, taxa de corrosão zero e absorção de água. CONCLUSÃO: Considerando as propriedades físicas e mecânicas do PEEK, esse material pode ser utilizado em prótese parcial removível e prótese parcial fixa. A literatura mostra em estudos in sílico e in vitro que o PEEK ainda possui características clinicas inferiores quando em comparação com implantes de titânio.

PALAVRAS-CHAVE: Bioatividade. Implante. Prótese.

\begin{abstract}
OBJECTIVE: To review the literature on the polyether etherketone biomaterial (PEEK) emphasizing the mechanical properties and applications in dentistry. MATERIAL AND METHOD: A search was performed in the electronic databases PubMed, Scielo and Bireme, using the search terms: Dental Materials, Dental Implants, Dental Prosthesis. The inclusion and exclusion criteria were studies in silico, in vitro, systematic review and literature that addresses the variables under study, language in English and Portuguese. RESULTS: PEEK has been gaining important prominence in dentistry, since it has a high resistance to weight ratio, elastic properties similar to human bone, ability to absorb shock during chewing, high wear resistance, low zero corrosion rate and absorption of water. CONCLUSION: The literature shows in silico and in vitro studies that PEEK still has inferior clinical characteristics when compared to titanium implants.
\end{abstract}

KEYWORDS: PEEK. Implants. Prosthodontics. 


\section{Introdução}

As reabilitações orais com metais estão cada vez mais entrando em decadência devido a fatores como o aumento dos requisitos estéticos do paciente e a legislação em alguns países devido à possível incompatibilidade do material relatada. Nos últimos anos têm ocorrido várias pesquisas com o intuito de encontrar materiais alternativos aos metais para reabilitações orais, com características físico-mecânicas adequadas e biologicamente compatíveis|?.

O poli-éter-éter-cetona (PEEK) devido as suas propriedades mecânicas e biológicas tem se mostrado um dos materiais alternativos mais promissores para uso em odontologia|. A unidade do monômero éter-étercetona é polimerizada através da reação da desalquilação pelo crescimento de bisfenolatos para formar a poli-éter-éter-cetona. Portanto o PEEK é um polímero aromático, linear, semicristalino, quimicamente inerte, pertencente a uma classe importante de termoplásticos de engenharia de alto desempenho, que atraiu a atenção de pesquisadores dentários devido as suas altas propriedades mecânicas e físicas, alto grau de estabilidade térmica e dimensiona佣.

Este material vem sendo utilizado no campo das cirurgias ortopédicas desde a década de 1980, incluindo placas de fixação de fratura, placas cranianas artificiais, componentes das articulações do dedo, joelho e da coluna vertebral. Na área odontológica, o PEEK foi introduzido em 1992, primeiro sob a forma de pilares e posteriormente como implantes].

Devido as suas características e imensa versatilidade, o PEEK é visto como um material protético que pode desempenhar múltiplos papéis em reabilitação orais. No campo odontológico, o PEEK vem sendo explorado como um material em várias aplicações, incluindo implantes dentários, pilares temporários para próteses implantadas, estruturas de prótese de protocolo Branemark, próteses parciais removíveis e próteses parciais fixas朋.
O objetivo do presente estudo é revisar a literatura sobre o biomaterial poli-éter-éter-cetona (PEEK) enfatizando as propriedades mecânicas e aplicações na odontologia.

\section{Material e métodos}

A literatura foi selecionada por meio de resumos e abstracts, utilizando as palavras-chaves buscadas no Mesh: Dental Materials, Dental Implants, Dental Prosthesis; sendo assim as variáveis a serem abordadas são aplicação do PEEK em prótese parcial fixa, removível, implantes e pilares. Os critérios de inclusão foram: revisão sistemática de literatura, estudo clínico, estudo in sílico, in vitro, literatura que abordassem as variáveis em estudo idioma em inglês e português. Os critérios de exclusão foram: carta ao editor, revisão de literatura e variáveis que não abordassem a presente revisão.

\section{Revisão da Literatura Discutida}

Em comparação com os materiais tradicionais utilizados em reabilitações orais, o PEEK apresenta grandes vantagens devido às suas excelentes propriedades (Quadro 1). Possui uma relação de alta resistência a peso, propriedades elásticas similares ao osso humano, capacidade de absorver choque durante a mastigação, alta resistência ao desgaste, taxa de corrosão zero e absorção de água extremamente baixal|.

Os anéis aromáticos fazem do PEEK uma estrutura química estável, resistente à degradação química e térmica, o que o torna atraente para uso médico, especialmente devido à sua capacidade de ser esterilizado por radiação e calor sem danos estruturaisf. 
Quadro 1. Material, elasticidade e resistência.

\begin{tabular}{|l|c|c|c|}
\hline \multicolumn{1}{|c|}{ Material } & $\begin{array}{c}\text { Módulo de } \\
\text { Elasticidade GPa) }\end{array}$ & $\begin{array}{c}\text { Resistência à } \\
\text { tração (MPa) }\end{array}$ & Referências \\
\hline PEEK & 3,6 & $90-100$ & (PEDRO, 2016) \\
\hline PEEK - RFC* & 18 & 120 & (NAJEEB et al., 2016) \\
\hline Cr-Co & $200-220$ & $600-700$ & (PEDRO, 2016) \\
\hline Titânio & $102-110$ & $954-976$ & (NAJEEB et al., 2016) \\
\hline Osso cortical & 14 & $104-121$ & (NAJEEB et al., 2016) \\
\hline Dentina & 15 & 104 & (NAJEEB et al., 2016) \\
\hline
\end{tabular}

*RFC - Reforçado por fibra de carbono.

Fonte: Os autores (2020).

\section{Aplicação do PEEK em Prótese Parcial Fixa}

Sinha et al. (2017), em sua experiência de utilizar PEEK como estrutura de próteses parciais fixas superior e inferior, mostrou resultados biológicos bastante satisfatórios com alto grau de conforto e aceitabilidade do paciente. Durante o acompanhamento do caso clínico por 6 meses observou pouco acúmulo de biofilme e tecido gengival saudável, evidenciando a natureza biocompatível do material.

Assim como outras restaurações sem metal, o PEEK possui baixa energia superficial e, além disso, natureza hidrofóbica, requerendo tratamento de superfície para se obter adesão das resinas e cimentos de união. No entanto, ainda não foi estabelecido um método eficiente para modificar a morfologia da superfície do PEEK. Por ser um polímero com estrutura química estável, é altamente resistente à degradação química mesmo em contato com ácidos fortes como o ácido fluorídrico a 9,5\%. Tornando assim difícil de estabelecer um protocolo para cimentar coroas únitárias ou próteses parciais fixas (PPF's) usando PEEK como infra-estrutura?.
Stawarczyk et al. (2015), investigou a influência de diferentes tratamentos de superfície no PEEK e sistemas adesivos sobre a carga de fratura de PPF's recobertas com compósito resinoso. Observaram que todas as PPF's apresentaram fissuras no material de revestimento independente do tratamento de superfície ou sistema adesivo utilizado, sem nenhuma fratura evidente das infraestruturas em PEEK.

Nazari et al., (2016), realizou um estudo com o intuito de comparar a resistência à fratura de PPF's suportadas por implantes confeccionadas com diferentes materiais, após receber carga axial compressiva. Três tipos de materiais foram utilizados como infraestrutura da PPF: zircônia (Zr), liga de níquel-cromo (Ni$\mathrm{Cr}$ ) e poli-éter-éter-cetona (PEEK). Sendo que os dois primeiros receberam cerâmica de cobertura (VITA VM9, Zahnfabrik, Bad Säckingen, Alemanha), e o último foi revestido com compósito resinoso (Crea.lign; Bredent GmbH \& Co KG, Senden, Alemanha), após receber tratamento de superfície com jato de óxido de alumínio e aplicação de sistema adesivo (Visio. link; Bredent GmbH \& Co KG, Senden, Alemanha). O resultado obtido foi que todas as PPF's em PEEK apresentaram falha adesiva (fratura na interface infraestrutura/compósito) após atingir a carga de fratura de $1430,47 \pm 262,21 \mathrm{~N}$, sugerindo a fraca resistência de união entre o PEEK e o material resinoso. As cargas de fratura dos grupos de $\mathrm{Zr}$ e Ni-Cr foram de 2086,31 $\pm 362,61,5591,74 \pm 1200,29$, respectivamente. 
Concluindo, portanto, que as restaurações com infraestrutura em $\mathrm{Ni}-\mathrm{Cr}$ e $\mathrm{Zr}$ apresentam resistência à fratura significativamente maior do que usando PEEK em restaurações. Apesar das restaurações PEEK terem mostrado resistência à fratura significativamente menor do que a dos outros materiais, os autores relataram que a aplicação como infraestrutura protética pode ser viável já que a resistência à compressão axial é maior do que a força de mordida máxima relatada na região molar em indivíduos com hábitos parafuncionais como o bruxismo, que varia entre 500 $\mathrm{N}$ a $880 \mathrm{~N}$. Portanto, as restaurações PEEK podem potencialmente suportar forças oclusais fisiológicas.

\section{Implantes e pilares de PEEK}

Em implantodontia, o titânio tem sido utilizado amplamente para a fabricação de implantes pilares e parafusos dos pilares, sendo considerado o material de escolha para o uso intraósseo no campo médico e odontológico, devido a características como a biocompatibilidade, osteointegração, resistência mecânica e a alta resistência à corrosão em um ambiente fisiológicol.

No entanto, em contrapartida ao sucesso dos implantes dentários de titânio, casos cada vez mais frequentes de hipersensibilidade e respostas alérgicas ao titânio e a suas ligas vem sendo relatados na literatura\}. Além disso, o titânio possui alto módulo de elasticidade, ao em vez do implante absorver parte das forças de mastigação, ele transfere toda a carga para o osso adjacente, causando forte tendência ao estresse. Esta sobrecarga oclusal torna o osso marginal periimplantar susceptível a reabsorção óssea e, consequentemente, eventual falha de próteses apoiadas por implantes?.

O parafuso do pilar, ou até mesmo o próprio implante, estão sujeitos a fraturas em situações associadas ao aperto inadequado e/ou afrouxamento dos parafusos. Além de outros fatores agravantes como ausência de assentamento passivo por desajuste cervical da prótese, princípios de oclusão imprópria, contatos oclusivos prematuros, hábitos parafuncionais, etc. O pilar quando fraturado pode ser substituído por outro. Entretanto, às vezes, o parafuso não pode ser removido e todo o implante deve ser substituído cirurgicamente ${ }^{4}$. Frente à esses problemas, sugeriu-se que novos materiais fossem testados para a fabricação de implantes dentários e componentes intermediários明.

Os materiais poliméricos ganharam foco em pesquisas devido à sua elevada capacidade de resiliência mecânica, resistência à fratura, absorção de choque e menor transmissão de forças ao osso adjacente. Tendo em vista que essas propriedades poderiam evitar fraturas do parafuso de pilar e a transmissão de sobrecargas oclusais para o osso marginal em torno de implantes dentários com subsequente perda óssea

O PEEK possui módulo de elasticidade semelhante ao do osso, com excelente capacidade de resiliência mecânica e absorção de choque. Portanto pilares e implantes dentários produzidos a partir desse polímero podem absorver e favorecer a dissipação das cargas mastigatórias para o osso periimplantar, que por sua vez pode evitar falhas de implantesf.

Neumann et al., (2014) compararam a resistência à fratura de parafusos de retenção de pilares fabricados de titânio, PEEK e PEEK reforçado por fibra de carbono (RFC) a 30\%, submetidos à força de compressão. Com os resultados obtidos, verificou-se que os parafusos de titânio apresentaram maior resistência à fratura, em comparação com parafusos de PEEK os de PEEK-RFC a 30\%. No mesmo estudo, não foi observado nenhuma diferença estatisticamente significante entre a resistência à fratura dos parafusos de PEEK e os de PEEK-RFC a 30\%. No entanto, pesquisas mostram que a adição de fibras de carbono a matriz PEEK aumenta significativamente a estabilidade dimensional, a dureza, a resistência à flexão e resistência mecânicaf.

Estudos sugerem que o PEEK de grau implantável possui capacidade de formação de osso comparável ao titânio usinado]. Entretanto, o PEEK sem reforço de biomateriais é quimicamente inerte e por ter propriedades de superfície hidrofóbicas, não é bom condutor de adesão celular, o que retarda o processo de osteointegração entre o osso e o implante PEEK. Pesquisas revelaram que a bioatividade do PEEK pode ser melhorada na modificação de sua superfície com titânioß.

Um estudo in vitro teve por objetivo analisar comparativamente o potencial osteogênico e a citotoxicidade entre o PEEK, PEEK revestido com dióxido de titânio (TiO2) e PEEK misturado com TiO2. Com a análise dos ensaios, observaram uma maior adesão e proliferação 
de células osteoblásticas com formação óssea no PEEK revestido com TiO2, seguido por amostras do PEEK misturado com TiO2, enquanto as amostras PEEK sem modificação apresentaram a menor capacidade de adesão e mineralização ósseaß.

A bioatividade do PEEK puro foi comparada com o nanocompósito PEEK, fabricado a parti da adição de nanopartículas de $\mathrm{TiO} 2$ (n-TiO2) a matriz PEEK, tanto in vitro como in vivo. $\mathrm{Na}$ análise dos estudos in vitro, verificaram que o $\mathrm{n}$-TiO2 melhorou significativamente a ligação e disseminação de osteoblastos na superfície do n-TiO2/PEEK em relação ao PEEK puro, concluindo que ao invés de causar citotoxicidade ou perturbar a progressão do ciclo celular, o n-TiO2 melhorou a bioatividade do PEEK. Os estudos in vivo foram realizados com a implantação de implantes PEEK puro e n-TiO2/PEEK na tíbia de cachorros. Após a análise de cortes histológicos e imagens tomográficas tridimensionais, observaram uma maior regeneração óssea em torno dos implantes de n-TiO2/PEEK, indicado por um maior volume de osso/tecido osteointegradol .

O módulo de elasticidade do osso e do PEEK são semelhantes, podendo assim ser utilizado em muitas áreas na odontologia, no entanto a biocompatibilidade dos implantes dentários com esse material ainda requer melhorias 1 .

\section{PEEK como infraestrutura de Prótese Parcial Removível}

O planejamento adequado da prótese parcial removível (PPR) e o material de escolha para a sua fabricação são essenciais para a sua retenção, estabilidade e suporte, principalmente em PPR de extremo livre, com ênfase no controle de força durante as forças compressivas, o que constitui um fator destrutivo para os dentes pilares e tecidos circunjacentes' 1 .

Devido aos princípios de biomecânica em arcos parcialmente edêntulos, a liga metálica de CromoCobalto $(\mathrm{Cr}-\mathrm{Co})$ é atualmente o material de eleição para a construção da infraestrutura desse tipo de prótese. No entanto, o mesmo apresenta algumas limitações, como: problemas estéticos, principalmente se os grampos se localizarem na zona anterior, desconforto sentido pelos pacientes, efeitos deletérios sobre os dentes pilares, fratura dos grampos e microporosidades formadas na sequência da sua fabricação devido à contração do material. Sendo que as microporosidades tanto da resina acrílica como da estrutura metálica aumenta a predisposição à proliferação de Candida Albicans e maior tendência para a formação de placa bacteriana na superfície da prótese e nos dentes em contatom.

Com o intuito de superar estas limitações descritas, tem sido proposto o uso do polímero PEEK, devido aos bons resultados apresentados na área ortopédica. Este polímero de alto desempenho tem ganhado foco na sua implantação na área da PPR pelo fato de ser um material biocompatível, não alergênico, mais estético, possuir menor peso, flexibilidade semelhante ao osso, boa resistência ao desgaste e bom polimento, oferecendo uma maior lisura superficial e menor adesão bacteriana!.

Pedro (2016), comparou PPR's confeccionadas com o polímero PEEK com a convencional PPR em $\mathrm{Cr}$ Co. No experimento foram confeccionadas próteses para substituição dos elementos 26 e 27 perdidos (desdentado maxilar Classe II de Kennedy sem áreas de modificação). A infraestrutura em PEEK confeccionada pelo método de injeção possuía a mesma geometria da infraestrutura em $\mathrm{Cr}$-Co. Para a análise deste estudo foi utilizado o método de Análise por Correlação de Imagem Digital 3D (CID-3D) (Correlated Solutions ${ }^{\circ}$, Columbia, USA). No estudo foram realizados ensaios experimentais para análise comparativa do deslocamento da sela distal livre e do deslocamento do conector maior das estruturas em $\mathrm{Cr}$-Co e PEEK. Em ambos ensaios, a infraestrutura em PEEK apresentou maiores micromovimentos de deslocamento tanto no teste de compressão vertical da sela como do teste de flexão do conector maior. A justificativa para tal resultado foi atribuído ao fato do menor módulo de elasticidade deste material (3,6 GPa) quando comparado com o Cr-Co (200-220 GPa) 11. No segundo ensaio também foi constatado que a estrutura em $\mathrm{Cr}$-Co quando submetido à força de tração apresentou um padrão de distribuição dos micromovimentos mais uniforme quando comparado à estrutura em PEEK, onde o padrão de concentração de deslocamentos na área do conector maior centrou-se em determinadas zonas desta estrutura 1 . 
Sendo assim numa zona de compressão dos tecidos nessa região, com potencial aumento da reabsorção óssea e agravamento do prognóstico da reabilitação. A rigidez do conector maior de uma PPR está diretamente relacionada ao fato de conectores mais rígidos diminuírem o deslocamento da PPR e, por consequência, a diminuição de forças nocivas sobre os dentes pilares, quando submetidos à carga oclusal. Do contrário, ocorrerá maiores danos ao periodonto de inseção, levando a uma maior reabsorção óssea e, consequentemente, a uma desadaptação da PPR com maior desconforto para paciente.

A literatura ainda é escassa na utilização de PEEK em próteses parciais removíveis, no entanto, a partir dos resultados obtidos a infraestrutura para PPR em PEEK ainda não reúne as condições adequadas para o seu uso clínico, uma vez que não demonstrou no estudo rigidez e estabilidade semelhante à estrutura metálica国.

\section{Conclusão}

A partir deste estudo, considerando as propriedades físicas e mecânicas do PEEK, esse material pode ser utilizado em prótese parcial removível e prótese parcial fixa. A literatura mostra em estudos in sílico e in vitro que o PEEK ainda possui características clinicas inferiores quando em comparação com implantes de titânio.

\section{Contribuições dos autores}

Galvão I, Carvalho P, Feitosa RS, Sousa El responsabilizaramse pela escrita do artigo. Grangeiro MTV e Figueiredo VMG responsabilizaram-se pela correção do artigo.

\section{Conflitos de interesses}

Nenhum conflito financeiro, legal ou político envolvendo terceiros (governo, empresas e fundações privadas, etc.) foi declarado para nenhum aspecto do trabalho submetido (incluindo, mas não se limitando a subvenções e financiamentos, participação em conselho consultivo, desenho de estudo, preparação de manuscrito, análise estatística, etc.).

\section{Referências}

1. Sinha N, Gupta N, Reddy KM, Shastry YM. Versatility of PEEK as a fixed partial denture framework. J Indian Prosthodont Soc. 2017;17(1):80-83. doi: 10.4103/0972-4052.197941

2. Rocha RFV, Anami LC, Campos TMB, Melo RM, Bottino MA Souza ROA. Bonding of the polymer polyetheretherketone (PEEK) to human dentin: effect of surface treatments. Braz Dent J. 2016;27(6):693-699. doi: 10.1590/0103-6440201600796

3. Nazari V, Ghodsi S, Alikhasi M, Sahebi M, Shamshiri AR. Fracture strength of three-unit implant supported fixed partial dentures with excessive crown height fabricated from different materials. J Dent. 2016;13(6):400-406.

4. Neumann EAF, Villar CC, França FMG. Fracture resistance of abutment screws made of titanium, polyetheretherketone, and carbon fiber-reinforced polyetheretherketone. Braz Oral Res. 2014;28(1):1-5. doi: 10.1590/1807-3107bor-2014.vol28.0028

5. Stock V, Schmidlin PR, Merk S, Wagner C, Roos M, Eichberger $M$ et al. PEEK primary crowns with cobalt-chromium, zirconia and galvanic secondary crowns with different tapers - A comparison of retention forces. Materials. 2016;9(3):187. doi: 10.3390/ ma9030187

6. Stawarczyk B, Thrun H, Eichberger M, Roos M, Edelhoff D, Schweiger J et al. Effect of different surface pretreatments and adhesives on the load-bearing capacity of veneered 3-unit PEEK FDPs. J Prosthet Dent. 2015;114(5):666-673. doi: 10.1016/j. prosdent.2015.06.006

7. Goutam M, Giriyapura C, Mishra SK, Gupta S. Titanium allergy: a literature review. Indian J Dermatol. 2014;59(6):630. doi: 10.4103/0019-5154.143526

8. Kumar TA, Jei JB, Muthukumar B. Comparison of osteogenic potential of poly-ether-ether-ketone with titanium-coated poly-ether-ether-ketone and titanium-blended poly-etherether-ketone: An in vitro study. J Indian Prosthodont Soc. 2017;17(2):167-174. doi: 10.4103/jips.jips_166_16

9. Wu X, Liu X, Wei J, Ma J, Deng F, Wei S. Nano-TiO2/PEEK bioactive composite as a bone substitute material: in vitro and in vivo studies. Int J Nanomedicine. 2012;7:1215-1225. doi: $\underline{10.2147 /}$ IJN.S28101

10. Najeeb S, Zafar MS, Khurshid Z, Siddiqui F. Applications of polyetheretherketone (PEEK) in oral implantology and prosthodontics. J Prosthodont Res. 2016;60(1):12-9. doi: 10.1016/j.jpor.2015.10.001

11. Pedro FJCDO. Análise de estruturas protéticas em PEEK pelo método de correlação de imagem digital tridimensional (CID-3D): um estudo piloto. [dissertação]. Portugal: Faculdade de Medicina da Universidade de Coimbra; 2016. 